\title{
COMPORTAMENTO INGESTIVO DE VACAS ALIMENTADAS COM CANA-DE-AÇÚCAR E DIFERENTES NÍVEIS DE CONCENTRADO\#
}

\author{
INTAKE BEHAVIOR OF COWS FED WITH SUGAR-CANE AND DIFFERENT \\ CONCENTRATE LEVELS
}

\author{
Costa, L.T. ${ }^{1 *}$, Silva, F.F. ${ }^{1 A}$, Veloso, C.M. ${ }^{1 \mathrm{~B}}$, Pires, A.J.V. ${ }^{1 \mathrm{C}}$, Rocha Neto, A.L. ${ }^{1 \mathrm{D}}$, Bonomo, P. ${ }^{1}$, \\ Mendes, F.B.L. ${ }^{1}$, Oliveira, J.S. ${ }^{1}$, Azevêdo, S.T. ${ }^{1}$ e Silva, V.L. ${ }^{1}$
}

${ }^{1}$ Universidade Estadual do Sudoeste da Bahia. Itapetinga. Brasil. *Itcosta80@yahoo.com.br; Affsilva@uesb.br; ${ }^{B} \mathrm{cmveloso@uesb.br;} \mathrm{'Aureliano@uesb.br;} \mathrm{Daires21@bol.com.br}$

\section{PaLAVRAS ChaVE ADICIONAIS \\ Ruminação. Suplementação. Eficiência alimentar.}

\section{RESUMO}

Foram estudados os efeitos de diferentes níveis de suplementação de vacas leiteiras alimentadas à base de cana-de-açúcar sobre o comportamento ingestivo destes animais. Os níveis de suplementação concentrada foram definidos pelo balanceamento das dietas para conter nutrientes suficientes para mantença e produção de 6, 9, 12 e $15 \mathrm{~kg}$ dia $^{-1}$ de leite. O experimento fo conduzido na fazenda Paulistinha, na cidade de Macarani-BA, no período de julho a setembro de 2006. Foram utilizadas 16 vacas mestiças Holandês $x$ Zebu distribuídas em um delineamento inteiramente ao acaso, onde estes animais foram observados por dois dias consecutivos e feito uma média dos resultados obtidos para cada animal, chegando a 32 observações e oito repetições por tratamento. Os tempos despendidos com alimentação, ruminação e ócio, o tempo de mastigação total, o número de bolos ruminais, o número de mastigações merícicas por dia e o tempo de ruminação por bolo não diferiram entre os tratamentos $(p>0,05)$, enquanto que para o número de mastigações merícicas por bolo apresentou um comportamento quadrático e para as eficiências de alimentação e ruminação houve um acréscimo linear com o aumento do nível de concentrado.

\#Projeto financiado pelo Banco do Nordeste e parte da dissertação do $1^{\circ}$ autor.

Recibido: 4-11-08. Aceptado: 15-9-09.

\section{AdDitional KEYWORDS}

Rumination. Supplementation. Feed efficiency.

\section{SUMMARY}

The effects of different levels of supplementation of dairy cows fed to the sugar-cane base on the intake behavior of these encourage were studied. The levels of concentrate supplementation were defined by the balance of the diets to contain sufficient nutrients for maintenance and production of $6,9,12$ and 15 kg.day ${ }^{-1}$ of milk. The experiment was driven in the Paulistinha farm, in Macarani - BA city, in the period of July to September of 2006. 16 cows crossbred Holstein x zebu used distributed in a design entirely at random, where these animals were observed by two consecutive days and made an average of the results obtained for each animal, arriving to 32 observations and eight repetitions for treatment. The times spent with feeding, rumination and leisure, the time of total mastication, the number of cakes ruminates, the number of mastications merícicas a day and the time of rumination for cake didn't differ among the treatments ( $p>0.05)$, while for the number of mastications merícicas for cake presented a quadratic behavior and for the feeding efficiencies and rumination had a lineal increment with the increase of the concentrate level.

\section{INTRODUÇÃO}

O estudo do comportamento ingestivo é uma ferramenta de grande importância na avaliação das dietas, possibilitando ajustar 
o manejo alimentar dos animais para obtenção de melhor desempenho produtivo. Segundo Dado e Allen (1995), o comportamento ingestivo do animal é constituído pelos tempos de alimentação, ruminação, ócio, eficiência de alimentação e ruminação.

O comportamento alimentar tem sido estudado com relação às características dos alimentos, à motilidade do pré-estômago, ao estado de vigília e ao ambiente climático. A diversidade de objetivos e condições experimentais conduziu a várias opções de técnicas de registro dos dados, na forma de observações visuais, registros semi-automáticos e automáticos e parâmetros estudados, selecionados para a descrição do comportamento ingestivo, como tempo de alimentação ou ruminação, número de alimentações, períodos de ruminação e eficiência de alimentação e ruminação (Dulphy et al., 1980; Forbes, 1995).

O desempenho animal é determinado pelo consumo de nutrientes, sua digestibilidade e metabolismo. O consumo de alimentos, por sua vez, pode ser influenciado por fatores ligados aos alimentos, como palatabilidade, textura e aparência visual, e fatores ligados aos animais, como estado emocional, interações e aprendizado (Mertens, 1994). O controle do consumo de alimentos está diretamente relacionado ao comportamento ingestivo (Chase et al., 1976). O consumo diário de alimentos compreende o número de refeições diárias, a sua duração e a taxa de ingestão. A vaca pode regular seu consumo diário de matéria seca (MS) (Dado e Allen, 1995; Grant e Albright, 1995) por meio do ajuste do número diário de refeições e do tamanho das mesmas (duração x taxa de ingestão).

Os ruminantes, como outras espécies, procuram ajustar o consumo alimentar às suas necessidades nutricionais, especialmente de energia (Arnold, 1985). Animais estabulados gastam em torno de uma hora consumindo alimentos ricos em energia, ou até mais de seis horas, para fontes com baixo teor de energia. Da mesma forma, o tempo despendido em ruminação é influenciado pela natureza da dieta e, provavelmente, é proporcional ao teor de parede celular dos volumosos. Assim, quanto maior a participação de alimentos volumosos na dieta, maior será o tempo despendido com ruminação (Van Soest, 1994). Alimentos concentrados e fenos finamente triturados ou peletizados reduzem o tempo de ruminação, enquanto volumosos com alto teor de parede celular tendem a aumentar o tempo de ruminação. $\mathrm{O}$ aumento do consumo tende a reduzir o tempo de ruminação por grama de alimento, fator provavelmente responsável pelo aumento de tamanho das partículas fecais, quando o consumo é elevado.

Para dietas volumosas, a mastigação aumenta a degradação ruminal, por elevar a MS e as frações de fibra potencialmente digerível e reduzir o tempo de latência de degradação da fibra. Para dietas de cereais, sabe-se que, quando grãos inteiros não sofrem influência da mastigação, a digestão é limitada, e, conseqüentemente, requerem processamento (Beauchemin, 1991).

Welch (1982) afirmou que o aumento no fornecimento de fibra indigestivel não incrementa a ruminação a mais de oito ou nove h.dia ${ }^{-1}$, sendo a eficácia de ruminação importante no controle da utilização de volumosos; assim, um animal que rumina mais volumoso durante esse período de tempo pode consumir mais e ser mais produtivo.

O tempo de ruminação é altamente correlacionado $(0,96)$ com o consumo de FDN em bovinos (Welch e Hooper, 1988). Albright (1993), em experimento com vacas, relatou resposta quadrática para os níveis de 26, 30 e 34\% de FDN nas dietas, com valores máximos estimados, respectivamente, do tempo despendido em ruminação e tempo total de mastigação de 344 e 558; 403 e $651 ; 414$ e 674 min.dia ${ }^{-1}$.

O presente trabalho foi conduzido com o objetivo de avaliar parâmetros do comportamento ingestivo de vacas em lactação alimentadas com dietas à base de 
INGESTÃO DE CANA-DE-AÇÚCAR E CONCENTRADO POR VACAS EM LACTAÇÃO

cana-de-açúcar com diferentes níveis de concentrado.

\section{MATERIAL E MÉTODOS}

O experimento foi conduzido na fazenda Paulistinha, na cidade de Macarani-BA, no período de julho a setembro de 2006. Foram utilizadas 16 vacas mestiças Holandês $\mathrm{x}$ Zebu (grau de sangue variando de $1 / 2$ a $3 / 4$ de sangue $\mathrm{H} \times \mathrm{Z}$ ), de terceira ou quarta lactação, com produção anterior entre 2500 e $3000 \mathrm{~kg}$, ajustada para 300 dias, manejadas a pasto na época das águas, e com 110 dias, em média, de lactação no início do período experimental.

Os quatro tratamentos foram constituídos de diferentes níveis de suplementação concentrada, tendo como volumoso a cana-de-açúcar (Saccharum officinarum), variedade RB 72-454, tratada com $1 \%$ de uma mistura de uréia e sulfato de amônia (9:1 partes), na fase experimental, antes disso foi realizado um período de adaptação, constituído de uma semana, no qual todos os animais foram alimentados com cana e $0,5 \%$ desta mistura. Os níveis de suplementação concentrada foram definidos pelo balanceamento das dietas para conter nutrientes suficientes para mantença e produção de 6, 9, 12 e $15 \mathrm{~kg}$. dia ${ }^{-1}$ de leite, de acordo com o NRC (2001), com base nos dados da análise bromatológica da cana-deaçúcar, previamente feita no início do período de adaptação. O teor de nutrientes digestíveis totais (NDT) da cana foi estimado a partir da equação de regressão NDT= $74,49-0,5635 * \mathrm{FDA}\left(\mathrm{r}^{2}=0,84\right)$, descrita por Cappelle et al. (2001), para volumosos.

As proporções estimadas dos ingredientes nos concentrados são apresentadas na tabela I, na base da matéria natural. A relação volumoso: concentrado foi de 100:0; 84:16; 76:24 e 70:30, na base da MS, para as dietas com produções estimadas de 6, 9, 12 e $15 \mathrm{~kg}$ de leite. dia $^{-1}$, respectivamente, sendo denominados de concentrados 1,2 e 3 para respectivas produções de 9,12 e $15 \mathrm{~kg}$ de leite. dia $^{-1}$.
Foi utilizado o delineamento inteiramente ao acaso, o que correspondeu a dois dias de observação, de 16 vacas, onde foi feito uma media dos valores encontrados, gerando oito repetições por tratamento e um total de 32 observações.

Os animais foram alojados em baias individuais, com um tamanho de quatro metros quadrados, providas de cochos e bebedouros automáticos. $\mathrm{O}$ alimento foi oferecido na forma de mistura completa, duas vezes ao dia, às 6 e às 15 horas, à vontade, de modo a permitir de 5 a $10 \%$ de sobras.

Os animais foram submetidos a períodos de observação visual, para avaliar o comportamento ingestivo, durante 24 horas. A coleta de dados para saber o tempo gasto nas atividades de alimentação, ruminação e ócio foi feita com o uso de cronômetros digitais, manuseados por quatro observadores, durante o período.

As atividades foram registradas a cada cinco minutos de intervalo, conforme recomendado por Gary et al. (1970). No dia seguinte, foi realizada a determinação do número de mastigações merícicas e do tempo

Tabela I. Proporção dos ingredientes nos concentrados, na base da matéria natural. (Proportion of the ingredients in concentrates, based on natural matter).

\begin{tabular}{lccc}
\hline & \multicolumn{3}{c}{ Concentrado } \\
Ingrediente & 1 & 2 & 3 \\
\hline Calcário calcítico (\%) & 0,60 & 0,83 & 0,18 \\
Farelo de soja (\%) & 19,15 & 23,98 & 21,44 \\
Fosfato bicálcico (\%) & 7,49 & 4,93 & 3,94 \\
Fubá de milho (\%) & 66,64 & 65,56 & 70,32 \\
Sal mineral $^{1}(\%)$ & 6,12 & 4,70 & 4,12 \\
\hline
\end{tabular}

${ }^{1}$ Composição: cálcio, $18,5 \%$; fósforo, $9 \%$; magnésio, $0,4 \%$; enxofre, $1 \%$; sódio, $11,7 \%$; selênio, 30 ppm; cobre, 1500 ppm; zinco, 4000 ppm; manganês, 1200 ppm; iodo, 150 ppm; cobalto, $150 \mathrm{ppm}$ 
Tabela II. Composição química da cana-de-açúcar e dos concentrados e seus respectivos desvios padrão. (Chemical composition of sugar cane and concentrates and their standard deviations).

\begin{tabular}{|c|c|c|c|c|c|c|c|c|}
\hline \multirow[t]{2}{*}{ Item } & \multirow[t]{2}{*}{ Volumoso } & \multirow[t]{2}{*}{$\mathrm{DP}$} & \multicolumn{6}{|c|}{ Concentrado } \\
\hline & & & 1 & $\mathrm{DP}$ & 2 & $\mathrm{DP}$ & 3 & $\mathrm{DP}$ \\
\hline MS \% & 27,2 & 2,8 & 86,3 & 0,8 & 87,0 & 1,6 & 87,1 & 1,9 \\
\hline $\mathrm{MO}^{1}$ & 95,6 & 0,6 & 91,7 & 2,7 & 92,3 & 1,9 & 92,6 & 2,5 \\
\hline $\mathrm{PB}^{1}$ & 8,2 & 0,6 & 17,4 & 2,4 & 17,7 & 0,6 & 17,1 & 0,3 \\
\hline $\mathrm{EE}^{1}$ & 1,8 & 0,6 & 3,4 & 0,8 & 3,6 & 0,4 & 3,6 & 0,4 \\
\hline $\mathrm{FDN}^{1}$ & 59,4 & 3,3 & 38,4 & 4,0 & 35,8 & 1,2 & 34,9 & 2,8 \\
\hline FDA $^{1}$ & 39,9 & 2,3 & 11,4 & 2,0 & 8,2 & 0,9 & 10,6 & 1,5 \\
\hline $\mathrm{CNF}^{1}$ & 29,0 & 1,9 & 34,3 & 1,5 & 37,8 & 3,9 & 38,8 & 3,7 \\
\hline $\mathrm{PIDN}^{2}$ & 2,1 & 0,3 & 10,2 & 0,8 & 9,9 & 0,7 & 12,0 & 0,6 \\
\hline PIDA $^{2}$ & 1,5 & 0,5 & 7,4 & 1,2 & 7,4 & 1,0 & 6,4 & 1,6 \\
\hline $\operatorname{Lig}^{1}$ & 6,9 & 0,5 & 2,8 & 0,3 & 2,9 & 0,5 & 3,0 & 0,1 \\
\hline $\mathrm{MM}^{1}$ & 4,4 & 0,6 & 8,4 & 2,7 & 7,7 & 1,9 & 7,4 & 2,5 \\
\hline
\end{tabular}

MS: matéria seca; MO: matéria orgânica; PB: proteína bruta; EE: extrato etéreo; MM: matéria mineral; FDN: fibra em detergente neutro; FDA: fibra em detergente ácido; CNF: carboidrato não fibrosos; PIDN: proteína insolúvel em detergente neutro; PIDA: proteína insolúvel em detergente ácido; LIG: lignina; MM: matéria mineral.

${ }^{1}$ Porcentagem da matéria seca; ${ }^{2}$ Porcentagem da proteína total; DP: desvio padrão.

despendido na ruminação de cada bolo ruminal, com a utilização de cronômetro digital. Para essa avaliação, foram feitas observações, de todos os animais do experimento, de três bolos ruminais, em três períodos diferentes do dia (10-12; 14-16 e 19-21 horas). Durante a observação noturna dos animais, o ambiente foi mantido com iluminação artificial.

A eficiência de alimentação (EAL), a eficiência de ruminação (ERU), o número de bolos ruminais por dia (NBR), o tempo de mastigação total por dia (TMT) e o número de mastigações merícicas por dia (NMM) foram obtidos segundo metodologia descrita por Bürger et al. (2000).

As análises de matéria seca (MS), matéria orgânica (MO), proteína bruta (PB), extrato etéreo (EE), fibra em detergente neutro $(\mathrm{FDN})$, fibra em detergente ácido (FDA), carboidratos não fibrosos $(\mathrm{CNF})$ proteína insolúvel em detergente neutro (PIDN), proteína insolúvel em detergente ácido (PIDA), lignina (Lig) e matéria mineral (MM) das dietas foram realizadas conforme Silva e Queiroz(2002).

$\mathrm{Na}$ tabela II, estão apresentados a composição do volumoso e dos concentrados e seus respectivos desvios-padrão (s).

A composição química das dietas está apresentada na tabela III.

Os dados de tempo gasto com alimentação, ruminação e ócio, os consumos de matéria seca e fibra em detergente neutro, as eficiências de alimentação e ruminação, o tempo de mastigação total, o número de bolos regurgitados por dia, o número de mastigações totais por dia, o número de mastigações por bolo e o tempo de ruminação por bolo foram avaliados por meio de análises de variância e de regressão, utilizando-se o Sistema de Análises Estatísticas e Genéticas - SAEG (Ribeiro Jr., 2001). Os modelos estatísticos foram escolhidos de acordo com a significância dos coeficientes de regressão, utilizando-se o teste $t$ em nível de $5 \%$, e de determinação $\left(\mathrm{R}^{2}\right)$, e com o fenômeno biológico estudado. 
INGESTÃO DE CANA-DE-AÇÚCARE CONCENTRADO POR VACAS EM LACTAÇÃO

Tabela III. Composição química das dietas. (Chemical composition of diets).

\begin{tabular}{lcccc}
\hline \multirow{2}{*}{ Item } & \multicolumn{4}{c}{ Nível de concentrado (\%) } \\
& 0 & 16 & 24 & 30 \\
\hline MS \% & 27,2 & 36,7 & 41,6 & 45,2 \\
MO $^{1}$ & 95,6 & 95,0 & 94,8 & 94,7 \\
PB $^{1}$ & 8,2 & 9,6 & 10,4 & 10,8 \\
EE$^{1}$ & 1,8 & 2,1 & 2,3 & 2,4 \\
FDN $^{1}$ & 59,4 & 56,1 & 53,8 & 52,1 \\
FDA $^{1}$ & 39,9 & 35,3 & 31,3 & 31,1 \\
CNF $^{1}$ & 29,0 & 29,8 & 31,1 & 31,9 \\
PIDN $^{2}$ & 2,1 & 3,4 & 4,0 & 5,1 \\
PIDA $^{2}$ & 1,5 & 2,4 & 2,9 & 3,0 \\
Lig $^{1}$ & 6,9 & 6,2 & 5,9 & 5,7 \\
MM $^{1}$ & 4,4 & 5,0 & 5,2 & 5,3 \\
\hline
\end{tabular}

MS: matéria seca; MO: matéria orgânica; PB: proteína bruta; EE: extrato etéreo; MM: matéria mineral; FDN: fibra em detergente neutro; FDA: fibra em detergente ácido; CNF: carboidrato não fibrosos; PIDN: proteína insolúvel em detergente neutro; PIDA: proteína insolúvel em detergente ácido; LIG: lignina; MM: matéria mineral.

${ }^{1}$ Porcentagem da matéria seca; ${ }^{2}$ Porcentagem da proteína total.

\section{RESULTADOSEDISCUSSÃO}

Na tabela II os níveis de FDN do concentrado são considerados altos, 38,36; 35,82 e $34,86 \%$, o que pode ser atribuído a uma possível contaminação do milho, provavelmente por sabugo.
Os dados relativos aos tempos médios despendidos com alimentação, ruminação e ócio e seus respectivos coeficientes de determinação $\left(\mathrm{R}^{2}\right)$ e variação $(\mathrm{CV})$ são apresentados na tabela IV.

Não houve diferença dos tempos médios despendidos com alimentação, ruminação e ócio, entre as dietas experimentais $(\mathrm{p}>0,05)$. Sendo esses iguais a, para os níveis de concentrado de $0,16,24$ e $30 \%, 6,08 ; 5,84$; 6,02 e 6,45 horas.dia ${ }^{-1}$ para alimentação; 8,$85 ; 8,70 ; 8,46$ e 8,24 horas.dia ${ }^{-1}$ para ruminação; e 9,13; 9,52; 9,58 e 9,36 horas.dia ${ }^{-1}$ para ócio. Resultados semelhantes foram encontrados por Mendonça et al. (2004), trabalhando com vacas leiteiras alimentadas com silagem de milho e cana-de-açúcar com diferentes relações concentrado: volumoso, tendo encontrado valores iguais a 5,01; 4,36 e 4,16 horas.dia-1 de alimentação, 8,$41 ; 7,76$ e 7,71 horas.dia ${ }^{-1}$ de ruminação e 10,$58 ; 11,88$ e 12,13 horas. dia ${ }^{-1}$ em ócio, para silagem de milho com relação volumoso:concentrado de 60:40 e cana-deaçúcar, com relação volumoso concentrado de 60:40 e 50:50 respectivamente, sendo estes valores diferentes apenas para silagem com relação para o parâmetro ócio. Isso demonstra que quando se utilizou o volumoso cana de açúcar, mesmo variando os níveis de concentrado na dieta, não houve variação nos tempos despendidos com alimentação ruminação e ócio.

Bürger et al. (2000), trabalhando com

Tabela IV. Médias e equações de regressão dos tempos despendidos em alimentação, ruminação e ócio em função do nível de concentrado da dieta e seus respectivos coeficientes de determinação $\left(R^{2}\right)$ e variação $(C V)$. (Medium and the regression equations of time spent on food, rumination and leisure depending on the level of concentrate in the diet and their coefficients of determination $\left(\mathrm{R}^{2}\right)$ and variation $\left.(\mathrm{CV})\right)$.

\begin{tabular}{|c|c|c|c|c|c|c|c|}
\hline \multirow[t]{2}{*}{ Item } & \multicolumn{4}{|c|}{ Nível de concentrado (\%) } & \multirow[t]{2}{*}{ Equação } & \multirow[t]{2}{*}{$\mathrm{R}^{2}$} & \multirow[t]{2}{*}{$\mathrm{CV}$} \\
\hline & 0 & 16 & 24 & 30 & & & \\
\hline Alimentação (h) & 6,08 & 5,84 & 6,02 & 6,45 & $Y=6,10$ & - & 17,2 \\
\hline Ruminação (h) & 8,85 & 8,70 & 8,46 & 8,24 & $Y=8,56$ & - & 17,6 \\
\hline Ócio $(\mathrm{h})$ & 9,13 & 9,52 & 9,58 & 9,36 & $Y=9,40$ & - & 9,1 \\
\hline
\end{tabular}


bezerros holandeses alimentados com dietas contendo diferentes níveis de concentrado, verificaram diminuição linear do tempo gasto com alimentação e ruminação e acréscimo, também linear, do tempo gasto em ócio. Os valores encontrados pelos autores, para dietas contendo $30,45,60,75 \mathrm{e}$ $90 \%$ de concentrado, foram 4,$92 ; 3,96 ; 3,29$;

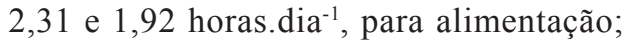
7,$52 ; 7,54 ; 6,83 ; 6,94$ e 4,23 horas. dia ${ }^{-1}$ para ruminação; e 10,$92 ; 11,69 ; 13,25 ; 14,02 \mathrm{e}$ 16,79 horas.dia ${ }^{-1}$ para ócio, diferentes dos encontrados no presente trabalho. Isso demonstra que a diferença de categoria influencia o comportamento dos animais, além dos autores terem trabalhado com alta porcentagem de concentrado na dieta.

Oliveira et al. (2007), trabalhando com vacas alimentadas à base de cana-de-açúcar, não encontraram diferença no comporta- mento das mesmas com relação a alimentação, ruminação e ócio, sendo os valores iguais a 4,1; 4,6 e 4,6; 7,3; 7,4 e 7,3; e 12,7; 12,0 e 12,5 horas.dia ${ }^{-1}$, para dietas com 0,25 e $50 \%$ de casca de café e casca de soja em substituição ao milho, respectivamente, em dietas com $60 \%$ de concentrado. O tempo médio encontrado por esses autores para alimentação e ruminação é menor que o observado no presente trabalho, o que pode ser atribuído à alta relação concentrado:volumoso, 60:40, utilizada pelos autores citados.

Pereira et al. (2007) encontraram diferença quanto ao tempo médio de alimentação, ruminação e ócio, trabalhando com novilhas alimentadas com dietas à base de capim-elefante com dois níveis de fibra em detergente neutro (FDN) 30 e $60 \%$. Sendo os tempos de alimentação e ruminação

Tabela $\boldsymbol{V}$. Valores médios e equações de regressão de consumo de MS (CMS), consumo de FDN (CFDN), eficiência de alimentação de MS (EAL) e de FDN (EALFDN), eficiência de ruminação de MS (ERU) e de FDN (ERUFDN), tempo de mastigação total (TMT), número de bolos ruminais (NBR), número de mastigações merícicas por dia (NMMd) e por bolo $(N M M b)$, e tempo de ruminação por bolo (TRB), em função dos níveis de concentrado na dieta. (Regression equations and average values for consumption of DM (CMS), consumption of FDN (CFDN), efficiency of supply of DM (EAL) and FDN (EALFDN), efficiency of rumination DM (ERU) and FDN (RUEFDN), total time of mastication (TMT), number of rumen boluses (NBR), number of mericic mastications per day (NMMd) and by bolus (NMMb), and rumination time by bolus (TRB), depending on the levels of concentrate in diet).

\begin{tabular}{|c|c|c|c|c|c|c|c|}
\hline \multirow[t]{2}{*}{ Item } & \multicolumn{4}{|c|}{ Nível de concentrado (\%) } & \multirow[t]{2}{*}{ Equação } & \multirow[t]{2}{*}{$\mathrm{R}^{2}$} & \multirow[t]{2}{*}{ CV } \\
\hline & 0 & 16 & 24 & 30 & & & \\
\hline $\mathrm{CMS}^{1}$ & 6,58 & 13,83 & 15,14 & 18,35 & $Y=0,3785 x+6,853$ & 0,98 & 18,12 \\
\hline $\mathrm{CFDN}^{1}$ & 3,13 & 5,37 & 6,53 & 7,51 & $Y=0,1449 x+3,0989$ & 0,99 & 13,73 \\
\hline$E A L^{2}$ & 1152,65 & 2380,61 & 2603,77 & 2869,88 & $Y=57,309 x+1248,8$ & 0,96 & 22,19 \\
\hline EALFDN $^{3}$ & 548,48 & 925,32 & 1123,16 & 1173,08 & $Y=21,652 x+563,6$ & 0,98 & 19,74 \\
\hline $\mathrm{ERU}^{2}$ & 739,41 & 1453,22 & 1584,31 & 2003,87 & $Y=39,895 x+747,04$ & 0,97 & 21,93 \\
\hline ERUFDN $^{3}$ & 351,84 & 564,05 & 683,41 & 820,15 & $Y=15,169 x+339,4$ & 0,99 & 20,212 \\
\hline $\mathrm{TMT}^{4}$ & 15,21 & 15,36 & 15,60 & 15,81 & $Y=15,50$ & - & 9,82 \\
\hline $\mathrm{NBR}^{5}$ & 704,47 & 598,99 & 687,44 & 672,81 & $Y=665,93$ & - & 18,31 \\
\hline NMMd $^{5}$ & 31805,79 & 35966,50 & 34565,85 & 34483,13 & $Y=34205,31$ & - & 11,13 \\
\hline $\mathrm{NMMb}^{6}$ & 47,13 & 61,25 & 51,50 & 51,75 & $Y=52,91$ & - & 12,20 \\
\hline $\mathrm{TRB}^{7}$ & 49,00 & 58,25 & 51,50 & 50,50 & $Y=52,31$ & - & 10,79 \\
\hline
\end{tabular}

${ }^{1} \mathrm{~kg} \cdot \mathrm{dia}^{-1} ;{ }^{2} \mathrm{~g} \mathrm{MS} \cdot \mathrm{h}^{-1} ;{ }^{3} \mathrm{~g}$ FDN. $\mathrm{h}^{-1} ;{ }^{4} \mathrm{~h} \cdot \mathrm{dia}^{-1} ;{ }^{5} \mathrm{n}^{0} \cdot \mathrm{dia}^{-1} ;{ }^{6} \mathrm{n}^{0} \cdot$ bolo-1 $^{-1} ;$ seg $\cdot$ bolo- ${ }^{-1}$. 
maiores quando se aumenta o teor de FDN da dieta em contrapartida ocorreu uma diminuição no tempo despendido com o ócio, demonstrando a importância deste ingrediente nas dietas em relação ao comportamento dos animais. O que diverge dos dados encontrados no presente trabalho, onde os tempos de alimentação e ruminação não diferenciaram isso pode ser explicado pela menor divergência no teor de FDN entre as dietas deste trabalho 59,$4 ; 56,1$; 53,8 e 52,1 para $0,16,24$ e $30 \%$ de concentrado nas dietas.

Os resultados referentes às médias de consumo de matéria seca (CMS), consumo de FDN (CFDN), eficiência de alimentação (EAL), eficiência de ruminação(ERU), tempo de mastigação total (TMT), número de bolos ruminais (NBR), número de mastigações merícicas (NMM) e tempo de ruminação por bolo ruminal (TRB) são apresentados na tabela $\mathrm{V}$

Houve acréscimo linear do consumo de MS e FDN $(p<0,05)$. Os consumos de MS e FDN aumentaram 0,38 e 0,14 kg para cada unidade de concentrado, chegando a 179 e $140 \%$ maiores com $30 \%$ de concentrado. Valores semelhantes de consumo de MS foram encontrados por Mendonça et al. (2004), 14,4 e 15,8 kg.dia-1 , para dietas à base de cana-de-açúcar, com relação volumoso: concentrado de 60:40 e 50:50, respectivamente, enquanto o consumo de FDN não diferenciou, possuindo valor médio de 4,45 $\mathrm{kg} \cdot \mathrm{dia}^{-1}$.

Resultados divergentes ao apresentado neste trabalho foram descritos por Bürger $e t$ al. (2000), encontrando comportamento quadrático do consumo de MS e linear decrescente do consumo de FDN. Isso pode ser explicado pelo alto nível de concentrado utilizado por esse autor.

A EAL, tanto de MS quanto de FDN, apresentou efeito linear crescente $(p<0,05)$, com resultados iguais a 1152,$65 ; 2380,61$; 2603,77 e 2869,88 g MS.h ${ }^{-1}$ e 548,48; 925,32; 1123,$16 ; 1173,08 \mathrm{~g} \mathrm{FDN}^{-h^{-1}}$ para os níveis de $0,16,24$ e $30 \%$ de concentrado na dieta. Isso indica que o nível de concentrado da dieta altera a EAL dos animais.

Resultados semelhantes de EAL foram encontrados por Bürger et al. (2000) e Mendonça et al. (2004). Entretanto, para EALFDN, foram encontrados efeito quadrático e ausência de efeito, com estimativa de valor máximo de 826,81 g FDN.h ${ }^{-1}$ para os respectivos autores, abaixo do valor máximo encontrado no presente trabalho. Isso pode ser explicado pela diferença de categoria utilizada, bezerros holandeses, por Bürger et al. (2000).

Pereira et al. (2007), trabalhando com diferentes teores de FDN na dieta, relatou que a EAL foi menor quando este teor foi maior, passando de 2980 para $2210 \mathrm{~g}$ de MS.h ${ }^{-1}$ quando se aumentou de 30 para $60 \%$ de FDN na dieta, valores estes parecidos com os encontrados no presente trabalho. Estes mesmos autores encontraram diferença quando comparados diferentes grupos genéticos, sendo menor para novilhas puras por cruza (PC), 2260; 2780 e $2750 \mathrm{~g}$ de MS.h ${ }^{-1}$ para PC, $15 / 16$ e 7/8 holandês/zebu, respectivamente, mostrando uma possível diferença quando se eleva o grau de sangue zebu.

A eficiência de ruminação da dieta, expressa em g MS.h ${ }^{-1}$ (ERU) e g FDN. ${ }^{-1}$ (ERUFDN), aumentou linearmente $(\mathrm{p}<0,05)$. Estes resultados podem ser explicados pela não divergência nos tempos gastos com ruminação e um maior consumo nas dietas com maiores níveis de concentrado.

Segundo Dulphy et al. (1980), a eficiência de ruminação aumenta quando o nível de concentrado da dieta é aumentado. Tal fato foi verificado no trabalho de Bürger et al. (2000), em que a ERU aumentou linearmente com a inclusão de concentrado nas dietas, enquanto a ERUFDN decresceu linearmente. Segundo os autores, isso ocorreu, provavelmente, em virtude do declínio na atividade celulolítica dos microrganismos ruminais, além de uma parte do concentrado ser regurgitada no bolo, durante a ruminação. Além disso, de acordo com Dado e Allen 
(1995), a maior taxa de ingestão de MS de dietas com baixa fibra está relacionada ao aumento na taxa de passagem da fibra nos compartimentos ruminais e, conseqüentemente, ao menor tempo de retenção ruminal da FDN.

Mendonça et al. (2004) não observaram diferença naERU, tanto expressa em g MS. $\mathrm{h}^{-1}$ quanto g FDN.h-1 ${ }^{-1}$, para dietas à base de silagem de milho e cana-de-açúcar, com diferentes níveis de concentrado, com valores iguais a 2136,$18 ; 1900,08$ e $2089,71 \mathrm{~g}$ MS.h $h^{-1}$ e 744,96; 587,54 e 582,19 para silagem de milho com relação volumoso:concentrado de 60:40 e cana-de-açúcar com relação volumoso:concentrado de 60:40 e 50:50, respectivamente.

O tempo de mastigação total (TMT) não variou entre as dietas $(\mathrm{p}>0,05)$, o que é explicado pela não variação de tempos de alimentação, ruminação e ócio do presente trabalho (tabela IV). Resultados semelhantes foram encontrados por Oliveira et al. (2007), trabalhando com vacas alimentadas à base de cana-de-açúcar, substituindo o milho por $25 \%$ de casca de café e $50 \%$ de casca de soja, encontrando valor médio igual a 11,60 h.dia ${ }^{-1}$, inferior ao valor médio encontrado no presente trabalho, 15,49h.dia ${ }^{-1}$. Isso pode ser atribuído à diferente relação volumoso:concentrado utilizada pelos autores, 40:60, e pelo alto teor de FDN das dietas do presente trabalho, com valor médio igual a 55,33\%, sendo que Pereira et al. (2007) observaram aumento no TMT quando se aumentou o teor de FDN das dietas.

O número de bolos ruminados (NBR) por dia não variou entre as dietas $(p>0,05)$. Estes dados corroboram com os encontrados por Mendonça et al. (2004), o que pode ser explicado pela não variação no TMT.

\section{BIBLIOGRAFIA}

Albright, J.L. 1993. Feeding behavior of dairy cattle. J. Dairy Sci., 76: 485-498.

Arnold, G.W. 1985. Ingestive behavior. Ethology of farm animals. In: Fraser, A.F. Elsevier.
Polli et al. (1996), trabalhando com bovinos e bubalinos alimentados com cana-deaçúcar ou silagem de milho, não encontraram diferenças quanto ao NBR. O NBR médio encontrado por estes autores foi inferior ao encontrado no presente estudo, 548 e 680 bolos ruminados, respectivamente, devido ao alto valor de TMT encontrado no presente trabalho.

O número de mastigações merícicas por dia (NMMd) e o tempo de ruminação por bolo (TRB) não variaram entre as dietas ( $\mathrm{p}>0,05)$. Polli et al. (1996) e Mendonça et al. (2004) também não encontraram diferença nestes parâmetros. Deswysen et al. (1987), citados por Mendonça et al. (2004), trabalhando com novilhas alimentadas com silagem de milho, constataram que os animais que consumiram mais alimentos apresentaram menor tempo de ruminação por bolo. Entretanto, no presente estudo, apesar do maior consumo da dieta com o maior nível de concentrado, não houve diferença $(p>0,05)$ no tempo de ruminação por bolo entre as dietas, assim como o número de mastigações merícicas por bolo (NMMb). Miranda et al. (1999), comparando uréia e cama-de-frango como fontes de nitrogênio não protéico para novilhas, verificaram que os animais alimentados com uréia mostraram tendência de maior NMMd, e de forma mais lenta.

\section{CONCLUSÃO}

Vacas leiteiras alimentadas com dietas à base de cana-de-açúcar, com diferentes níveis de concentrado, não apresentam diferenças quanto ao tempo despendido em alimentação, ruminação e ócio, mas como o aumento dos níveis de concentrado elevaram os consumos de MS e de FDN as eficiências alimentares foram beneficiadas.

Amsterdam. 186 pp.

Beauchemin, K.A. 1991.Effects of dietary neutral detergent fiber concentration and alfalfa hay quality on chewing, rumen function, and milk 


\section{INGESTÃO DE CANA-DE-AÇÚCARE CONCENTRADO POR VACAS EM LACTAÇÃO}

production of dairy cows. J. Dairy Sci., 74: 3140-3151.

Bürger, P.J., Pereira, J.C., Queiroz, A.C., Silva, J.F.C., Valadares Filho, S.C., Cecon,P.R. e Casali, A.D.P. 2000.Comportamento ingestivo em bezerros holandeses alimentados com dietas contendo diferentes níveis de concentrado. Rev. Bras Zootecn., 29: 236-242.

Cappelle, E.R., Valadares Filho, S.C., Silva, J.F.C. e Cecon, P.R. 2001.Estimativas do valor energético a partir de características químicas e bromatológicas dos alimentos. Rev. Bras Zootecn., 30: 1837-1856.

Chase, L.J., Wangsness, P.J. and Baumgardt, B.R. 1976. Feeding behavior of steers fed a complete mixed ration. J. Dairy Sci., 59: 1923-1928.

Dado, R.G. and Allen, M.S. 1995. Intake limitations, feeding behavior, and rumen function of cows challenged with rumen fill from dietary fiber or inert bulk. J. Dairy Sci., 78: 118-133.

Deswysen, A.G., Ellis, W.C. and Pond, K.R. 1987. Interrelationships among voluntary intake, eating and ruminating behavior and ruminal motility of heifers fed corn silage. J. Anim. Sci., 64: 835841.

Dulphy, J.P., Remond, B. and Theriez, M. 1980. Ingestive behavior and related activities in ruminants. In: Ruckebush, Y., Thivend, P. Digestive physiology and metabolism in ruminants. MTP. Lancaster. pp. 103-122.

Forbes, J.M. 1995. Voluntary food intake and diet selection in farm animals. CAB. Wallingford. $532 \mathrm{pp}$.

Gary, L.A., Sherritt, G.W. and Hale, E.B. 1970. Behavior of Charolais cattle on pasture. $J$. Anim. Sci., 30: 303-306.

Grant, R.J. and Albright, J.L. 1995.Feeding behavior and management factors during the transition period in dairy cattle. J. Anim. Sci., 73: 27912803.

Mendonça, S.S., Campos, J.M.S., Valadares Filho, S.C., Valadares, R.F.D., Soarez, C.A., Lana, R.P., Queiroz, A.C., Assis, A.J. e Pereira, M.L.A. 2004. Comportamento ingestivo de vacas leiteiras alimentadas com dietas à base de cana-de-açúcar ou silagem de milho. Rev. Bras. Zootecn., 33: 723-728.
Mertens, D.R. 1994. Regulation of forage intake. In Fahey, Jr. (Ed.). Forage quality, evaluation and utilization. American Society of Agronomy. Madison. pp. 450-493.

Miranda, L.F., Queiroz, A.C., Valadares Filho, S.C., Cecon, P.R., Pereira, E.S., Paulino, M.F. Lana, R.P. e Miranda, J.R. 1999. Comportamento ingestivo de novilhas leiteiras alimentadas com dietas à base de cana-de-açúcar. Rev. Bras. Zootecn., 28: 614-620.

NRC. 2001. National Research Council. Nutrient requirements of dairy cattle. $7^{\text {th }}$ ed. National Academy Press. Washington, D.C. 381 pp.

Oliveira, A.S., Campos, J.M.S., Valadares Filho, S.C., Assis, A.J., Teixeira, R.M.A., Rennó, L.N., Pina, D.S. e Oliveira, G.S. 2007. Substituição do milho pela casca de café ou de soja em dietas para vacas leiteiras: comportamento ingestivo, concentração de nitrogênio uréico no plasma e no leite, balanço de compostos nitrogenados e produção de proteína microbiana. Rev. Bras. Zootecn., 36: 205-215.

Pereira, J.C., Cunha, D.N.F.V., Cecon, P.R. e Faria, E.S. 2007. Comportamento Ingestivo e taxa de passagem de partículas em novilhas leiteiras de diferentes grupos genéticos submetidas a dietas com diferentes níveis de fibra. Rev. Bras. Zootecn., 36: 2134-2142.

Polli, V.A., Restle, J., Senna, D.B. e Almeida, S.R.S. 1996. Aspectos relativos à ruminação de bovinos e bubalinos em regime de confinamento. Rev. Soc. Bras. Zootecn., 25: 987-993.

Ribeiro Jr., J.I. 2001. Análises estatísticas no SAEG: Sistema de análises estatísticas. UFV. Viçosa-MG. $301 \mathrm{pp}$.

Silva, D.J. e Queiroz, A.C. 2002. Análise de alimentos: métodos químicos e biológicos. $2^{\mathrm{a}} \mathrm{ed}$. MG: Editora UFV. Viçosa. 165 pp.

Van Soest, P.J. 1994. Nutritional ecology of the ruminant. $2^{\mathrm{a}}$ ed. Cornell. Ithaca. $476 \mathrm{pp}$.

Welch, J.G. 1982. Rumination, particle size and passage from the rumen. J. Anim. Sci., 54: 885894.

Welch, J.G. and Hooper, A.P. 1988. Ingestion of feed and water. In: Church, D.C. (Ed). The ruminant animal: digestive physiology and nutrition. Reston. Englewood Cliffs. pp. 108-116. 